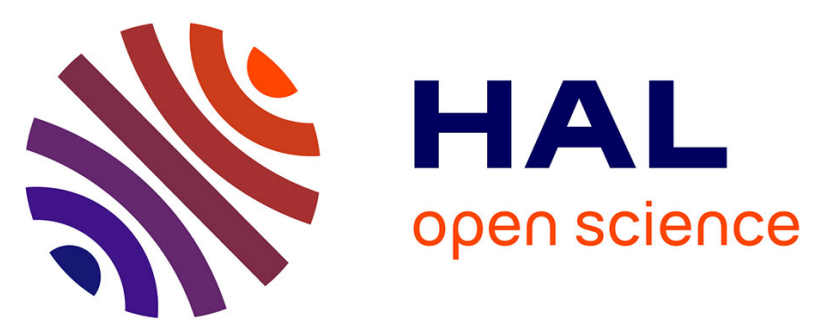

\title{
L'insémination artificielle de la truite (Salmo Gairdneri Richardson). III. Définition de la nature et de la molarité du tampon à employer avec les dilueurs d'insémination et de conservation.
}

Roland Billard, Bernard Jalabert, Bernard Breton, Anne-Marie Escaffre, M. Carpentier

\section{To cite this version:}

Roland Billard, Bernard Jalabert, Bernard Breton, Anne-Marie Escaffre, M. Carpentier. L'insémination artificielle de la truite (Salmo Gairdneri Richardson). III. Définition de la nature et de la molarité du tampon à employer avec les dilueurs d'insémination et de conservation.. Annales de biologie animale, biochimie, biophysique, 1974, 14 (4A), pp.611-621. 10.1051/rnd:19740404 . hal-00896906

\section{HAL Id: hal-00896906 https://hal.science/hal-00896906}

Submitted on 1 Jan 1974

HAL is a multi-disciplinary open access archive for the deposit and dissemination of scientific research documents, whether they are published or not. The documents may come from teaching and research institutions in France or abroad, or from public or private research centers.
L'archive ouverte pluridisciplinaire HAL, est destinée au dépôt et à la diffusion de documents scientifiques de niveau recherche, publiés ou non, émanant des établissements d'enseignement et de recherche français ou étrangers, des laboratoires publics ou privés. 
Ann. Biol. anim. Bioch. Biophys., I974, 14 (4-A), 6r I-62r.

\title{
L'INSÉMINATION ARTIFICIELLE DE LA TRUITE (SALMO GAIRDNERI RICHARDSON)
}

\section{III. - DÉFINITION DE LA NATURE ET DE LA MOLARITÉ DU TAMPON A EMPLOYER AVEC LES DILUEURS D'INSÉMINATION ET DE CONSERVATION}

\author{
R. BILLARD, B. JALABERT et B. BRETON
}

avec la collaboration technique de Anne-Marie Escaffre et M. Carpentier

Laboratoive de Physiologie des Poissons,

Centre national de Recherches zootechniques, I. N. R. A., 78350 Jouy en Josas

\section{RÉSUMÉ}

Des expériences ont été conduites dans le but de définir la nature et la molarité du tampon à utiliser avec les deux dilueurs d'insémination et de conservation définis précédemment. Les effets des différents tampons ont été testés d'une part sur la stabilité du pH du dilueur après introduction de quantités croissantes de $\mathrm{NaOH}$ et $\mathrm{HCl}$, de liquide colomique et d'ovules et d'autre part sur le pourcentage de fécondation établi sur de petits lots de 200 ovules. Les conclusions sont les suivantes :

I $^{\circ}$ Le dilueur d'insémination (solution de $\mathrm{NaCl}$ à 250 mosm) peut être avantageusement tamponné avec un tampon carbonate-bicarbonate $0,02 \mathrm{M}, \mathrm{pH}$ de 9,2 à 9,5. Ce tampon se révèle plus stable après introduction de grande quantité d'ovules que les autres tampons testés (Tris$\mathrm{HCl}$, Glycocolle- $\mathrm{NaOH}$ ), mais le pourcentage de fécondation reste le même avec chacun de ces tampons.

$2^{0}$ Lorsque le milieu minéral de liquide séminal (MMLS) est utilisé comme dilueur de conservation, la conservation du pouvoir fécondant du-spernest meilleure si le tampon utilisé est le Tris- $\mathrm{HCl}$; le $\mathrm{pH}$ optimum est voisin de 9,0 .

\section{INTRODUCTION}

Au cours de précédents travaux (Billard et al., I973; PetiT et al., I973), nous avons montré que l'emploi de dilueurs d'insémination ou de conservation permettait d'augmenter le pourcentage de fécondation des œufs de Truites, tout en réduisant 
le volume de sperme utilisé. Le pH s'est avéré être un facteur important. La présente étude a été conduite dans le but de définir la nature et la molarité du tampon à utiliser avec les différents dilueurs.

\section{MATÉRIEL, ET MÉTHODE}

Le matériel animal et les conditions d'expérimentation en petits lots ont été décrits précédemment (PETIT et al., 1973). Les dilueurs suivants ont été testés :

- une solution minérale reconstituant la composition minérale du liquide séminal (MMLS) qui

a été définie précédemment comme dilueur de conservation (BILlaRD et JALABERT, 1974);

- une solution tamponnée de $\mathrm{NaCl}$ (Prolabo RP) dite dilueur d'insémination.

Ces deux types de dilueurs ont été ajustés ou tamponnés avec 4 tampons de nature différente :

Tris-HCl selon GoMori (I955), carbonate-bicarbonate selon DeLoRY et KING (I945), glycocolle$\mathrm{NaOH}$ selon Sörensen (I909) et borate-NaOH rapporté par KOHLER (197I).

Dans tous les cas, la pression osmotique finale est d'environ $25^{\circ}$ milliosmoles. La stabilité $\mathrm{du} \mathrm{pH}$ du dilueur après addition des ovules et du sperme lors de la fécondation pose un certain nombre de problèmes. En effet, les ovules autour desquels il subsiste toujours un peu de liquide cœelomique dont le $\mathrm{pH}$ propre est de l'ordre de 8, entrainent une chute du $\mathrm{pH}$ du mélange. Une stabilisation de ce $\mathrm{pH}$ a été tentée, d'une part en portant la molarité du tampon de o,oz $\mathrm{M}$ à $\mathrm{o}$, I M et d'autre part en augmentant le volume du dilueur utilisé lors de l'insémination : $20 \mathrm{ml}$ au lieu de ro.

La stabilité du pH du dilueur d'insémination tamponné par différents tampons a été testée en ajoutant d'une part des quantités croissantes de $\mathrm{NaOH}$ et $\mathrm{HCl}(0,02 \mathrm{M})$ et d'autre part du liquide coelomique ou des ovules.

Les effets du $\mathrm{pH}$ et des tampons ont été étudiés, à la fois sur le pourcentage de fécondation et la conservation de la fertilité des gamètes dans les mêmes conditions que précédemment (BILLARD et JALABERT, I974).

\section{RÉSULTATS}

A. - Définition des caractéristiques optimum

( $\mathrm{pH}$, nature et molarité du tampon) des dilueurs

\section{Dilueur d'insémination.}

Nature du tampon.

Avec les systèmes Tris- $\mathrm{HCl}$, carbonate-bicarbonate et glycocolle- $\mathrm{NaOH}$, les pourcentages de fécondation obtenus dans l'expérience rapportée dans le tableau I, sont supérieurs à $85 \mathrm{p}$. Ioo pour des $\mathrm{pH}$ se situant aux alentours de 9,o. Avec le système borate- $\mathrm{NaOH}$, les résultats sont légèrement plus faibles.

Avec les 4 tampons, le $\mathrm{pH}$ du dilueur après insémination (c'est-à-dire après addition de 200 ovules et de Io ou I $\mu 1$ de sperme dans ro m1 de dilueur) s'est trouvé légèrement diminué.

\section{Molarité du tampon (tabl. 2).}

Lorsque la molarité du tampon ajouté au dilueur est augmentée (passage de $0,02 \mathrm{M}$ à $\mathrm{O}, \mathrm{I} \mathrm{M}$ ), la stabilité du $\mathrm{pH}$ du milieu après insémination est améliorée, mais le pourcentage de fécondation s'est trouvé légèrement diminué, surtout pour les $\mathrm{pH}$ supérieurs à 9,4 - sauf dans le cas du milieu tamponné par le Tris-HCl. 
TABLEAU I

Effets du pH et de la nature du tampon ajouté au dilueur d'insémination sur le pourcentage de fécondation d'ovules de Truite arc-en-ciel

(dilueur : solution de $\mathrm{NaCl}$ à $25^{\circ}$ milliosmoles)

\begin{tabular}{|c|c|c|c|c|}
\hline \multirow[t]{2}{*}{ Tampon $(0,02 \mathrm{M})$} & \multicolumn{2}{|c|}{$\mathrm{pH}$ du dilueur } & \multicolumn{2}{|c|}{$\begin{array}{l}\text { P. } 100 \text { de fécondation } \\
\text { selon dilution } \\
\text { du sperme }\end{array}$} \\
\hline & initial & $\begin{array}{l}\text { après insé- } \\
\text { mination }\end{array}$ & $10^{-4}(1)$ & $10^{-3}\left({ }^{1}\right)$ \\
\hline Tris- $\mathrm{HCl}$ & $\begin{array}{l}8,40 \\
8,65 \\
8,90 \\
9,10 \\
9,20\end{array}$ & $\begin{array}{l}8,40 \\
8,70 \\
8,80 \\
8,88 \\
9,00\end{array}$ & $\begin{array}{l}75,7 \\
89,9 \\
78,2 \\
88,2 \\
79,8\end{array}$ & $\begin{array}{l}67,4 \\
81,5 \\
84,5 \\
87,4 \\
88,2\end{array}$ \\
\hline Carbonate-bicarbonate & $\begin{array}{l}9,00 \\
9,18 \\
9,40 \\
9,65 \\
9,90\end{array}$ & $\begin{array}{l}8,85 \\
9,00 \\
9,35 \\
9,60 \\
9,80\end{array}$ & $\begin{array}{l}87,0 \\
86,0 \\
79,8 \\
57,1 \\
34,0\end{array}$ & $\begin{array}{l}79,6 \\
82,5 \\
82,4 \\
68,0 \\
63,1\end{array}$ \\
\hline Glycocolle- $\mathrm{NaOH}$ & $\begin{array}{l}8,15 \\
8,30 \\
8,66 \\
8,85 \\
9,00 \\
9,10 \\
9,55\end{array}$ & $\begin{array}{l}8,10 \\
8,20 \\
8,35 \\
8,45 \\
8,55 \\
8,60 \\
9,25\end{array}$ & $\begin{array}{c}60,3 \\
78,1 \\
86,5 \\
82,3 \\
- \\
81,2 \\
82,2\end{array}$ & $\begin{array}{l}82,3 \\
73,7 \\
86,1 \\
83,1 \\
85,5 \\
85,9 \\
81,3\end{array}$ \\
\hline Borate- $\mathrm{NaOH}$ & $\begin{array}{l}8,20 \\
8,35 \\
8,50 \\
8,80 \\
8,95 \\
9,25 \\
9,55\end{array}$ & $\begin{array}{l}8,10 \\
8,20 \\
8,30 \\
8,55 \\
8,60 \\
8,85 \\
9,15\end{array}$ & $\begin{array}{l}76,9 \\
64,0 \\
80,5 \\
56,8 \\
70,3 \\
56,4 \\
59,9\end{array}$ & $\begin{array}{l}73,8 \\
79,3 \\
58,5 \\
76,0 \\
78,7 \\
84,5 \\
76,7\end{array}$ \\
\hline $\begin{array}{l}\text { Témoin (sperme non } \\
\text { dilué) "Méthode sèche " }\end{array}$ & \multicolumn{2}{|c|}{$\begin{array}{l}\text { Début expérience } \\
\text { Fin expérience }\end{array}$} & \multicolumn{2}{|c|}{$\begin{array}{l}82,6 \\
74,5\end{array}$} \\
\hline
\end{tabular}

(1) Taux de dilution du sperme après mélange avec le dilueur. 


\section{TABLEAU 2}

Effets du pH et de la molarité du tampon du dilueur d'insémination sur le pourcentage de fécondation d'ovules de Truite arc-en-ciel

(dilueur : solution de $\mathrm{NaCl}$ à $25^{\circ}$ milliosmoles)

Expériences I et 2 : répétition avec 2 lots de femelles et 2 mâles différents

\begin{tabular}{|c|c|c|c|c|c|c|c|}
\hline \multirow{4}{*}{ Tampon } & \multirow{4}{*}{ Molarité } & \multicolumn{3}{|c|}{ Expérience $n^{0} 1$} & \multicolumn{3}{|c|}{ Expérience $n^{0} 2$} \\
\hline & & \multicolumn{2}{|c|}{$\begin{array}{l}\mathrm{pH} \text { du dilueur } \\
\text { tamponné }\end{array}$} & \multirow{3}{*}{$\begin{array}{c}\text { p. } 100 \mathrm{de} \\
\text { fécondation } \\
\text { (dilution } \\
\text { sperme : } \\
10^{-3} \text { ) }\end{array}$} & \multirow{3}{*}{$\underset{\text { initial }}{\mathrm{pH}}$} & \multirow{2}{*}{\multicolumn{2}{|c|}{$\begin{array}{l}\text { p. } 100 \text { de fécondation } \\
\text { selon dilution } \\
\text { du sperme }\end{array}$}} \\
\hline & & \multirow[b]{2}{*}{ initial } & \multirow{2}{*}{$\begin{array}{l}\text { après } \\
\text { mélange } \\
\text { avec les } \\
\text { ovules }\end{array}$} & & & & \\
\hline & & & & & & $10^{-4}\left({ }^{1}\right)$ & $10^{-3}(1)$ \\
\hline Glycocolle & 0,02 & $\begin{array}{l}8,2 \\
8,6 \\
9,0 \\
9,4 \\
9,75\end{array}$ & $\begin{array}{l}8,0 \\
8,45 \\
8,80 \\
9,15 \\
9,40\end{array}$ & $\begin{array}{l}86,0 \\
87,6 \\
83,0 \\
88,5 \\
84,5\end{array}$ & $\begin{array}{l}8,1 \\
8,6 \\
9,0 \\
9,5 \\
9,75\end{array}$ & $\begin{array}{l}71,3 \\
58,0 \\
49,5 \\
62,5 \\
46,8\end{array}$ & $\begin{array}{l}51,0 \\
65,8 \\
62,6 \\
58,4 \\
59,3\end{array}$ \\
\hline $\begin{array}{l}\text { Carbonate- } \\
\text { bicarbonate }\end{array}$ & 0,02 & $\begin{array}{r}8,8 \\
9,3 \\
9,8 \\
10,1\end{array}$ & $\begin{array}{l}8,50 \\
9,05 \\
9,55 \\
9,85\end{array}$ & $\begin{array}{l}86,0 \\
86,6 \\
77,2 \\
78,7\end{array}$ & $\begin{array}{r}8,7 \\
9,25 \\
9,75 \\
10,15\end{array}$ & $\begin{array}{l}57,8 \\
62,6 \\
58,7 \\
32,3\end{array}$ & $\begin{array}{l}63,2 \\
64,2 \\
64,0 \\
49,3\end{array}$ \\
\hline Tris- $\mathrm{HCl}$ & 0,02 & $\begin{array}{l}8,7 \\
9,0 \\
9,45\end{array}$ & $\begin{array}{l}8,50 \\
8,90 \\
9,30\end{array}$ & $\begin{array}{l}81,0 \\
81,3 \\
79,7\end{array}$ & $\begin{array}{l}8,7 \\
9,0 \\
9,5\end{array}$ & $\begin{array}{l}69,9 \\
66,5 \\
52,1\end{array}$ & $\begin{array}{l}65,2 \\
60,9 \\
59,9\end{array}$ \\
\hline Glycocolle & 0,1 & $\begin{array}{c}8,3 \\
8,8 \\
9,25 \\
9,7 \\
10,1\end{array}$ & $\begin{array}{r}8,25 \\
8,77 \\
9,10 \\
9,65 \\
10,00\end{array}$ & $\begin{array}{l}83,8 \\
90,1 \\
81,3 \\
58,8 \\
34,2\end{array}$ & $\begin{array}{r}8,3 \\
8,9 \\
9,2 \\
9,7 \\
10,0\end{array}$ & $\begin{array}{r}75,0 \\
71,8 \\
56,9 \\
25,0 \\
2,5\end{array}$ & $\begin{array}{l}62,0 \\
58,5 \\
54,4 \\
30,7 \\
18,9\end{array}$ \\
\hline $\begin{array}{l}\text { Carbonate- } \\
\text { bicarbonate }\end{array}$ & 0,1 & $\begin{array}{c}8,9 \\
9,4 \\
9,95 \\
10,4\end{array}$ & $\begin{array}{r}8,97 \\
9,40 \\
9,98 \\
10,28\end{array}$ & $\begin{array}{r}78,3 \\
82,3 \\
53,1 \\
4,5\end{array}$ & $\begin{array}{l}9,0 \\
9,4_{4} \\
9,95 \\
10,4\end{array}$ & $\begin{array}{c}64,1 \\
45,6 \\
14,6 \\
0\end{array}$ & $\begin{array}{r}58,8 \\
54,7 \\
16,7 \\
2,0\end{array}$ \\
\hline Tris-HCl & 0,1 & $\begin{array}{l}8,6 \\
9,05 \\
9,55\end{array}$ & $\begin{array}{l}8,55 \\
9,00 \\
9,40\end{array}$ & $\begin{array}{l}84,0 \\
82,9 \\
80,0\end{array}$ & $\begin{array}{l}8,5 \\
9,0 \\
9,55\end{array}$ & $\begin{array}{l}71,3 \\
65,1 \\
56,4\end{array}$ & $\begin{array}{l}74,0 \\
69,9 \\
67,4_{4}\end{array}$ \\
\hline $\begin{array}{l}\text { Témoin (sperme } \\
\text { non dilué) } \\
\text { "Méthode sèche" }\end{array}$ & & 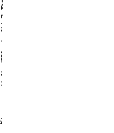 & & 75,6 & & & \\
\hline
\end{tabular}

(1) Taux de dilution final du sperme après mélange avec le dilueur. 
Effets de l'augmentation du volume de dilueur sur le pourcentage de fécondation (fig. I).

Le doublement du volume du dilueur qui atténue la chute de $\mathrm{pH}$ sans modification de la molarité du tampon entraîne une légère élévation du pourcentage de fécondation, parfois significative pour une dilution finale du sperme de $\mathrm{ro}^{-4}$.
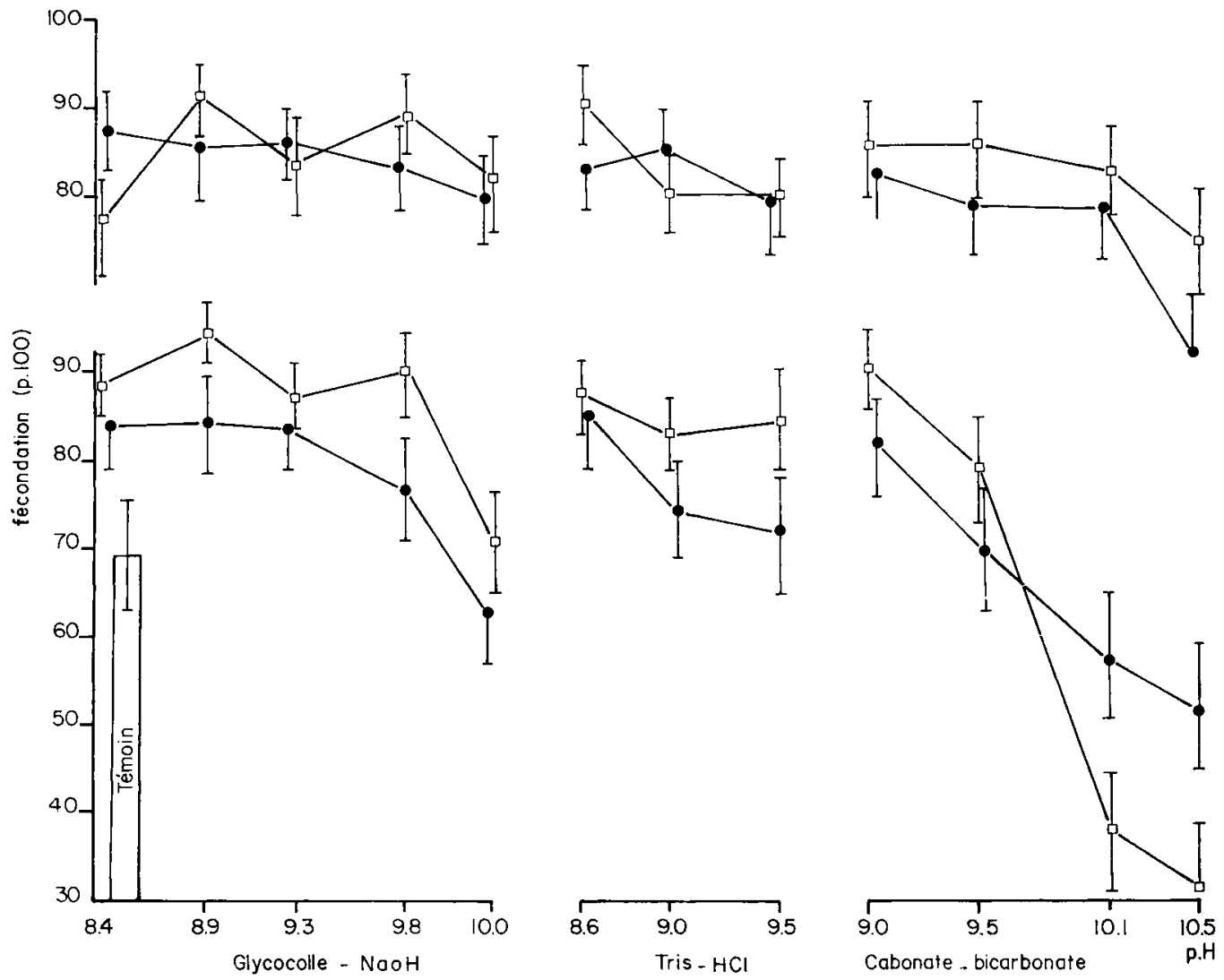

Fig. $1 .-$ Effets de la nature du tampon et du $p H$ du dilueur sur le pourcentage de fécondation

Dilution finale du sperme : en haut $\mathrm{IO}^{-3}$; en bas $\mathrm{IO}^{-4}$. Volume de dilueur ajouté pour chaque lot d'environ 200 ovules : $\bullet$ Io $\mathrm{ml} ; \square 20 \mathrm{ml}$.

Dilueur : solution de $\mathrm{NaCl}$ posm 250 mosm.

Les limites de l'intervalle de confiance d'une proportion (intervalle de confiance 95 p. I00) sont figurées sur les graphiques.

\section{Dilueur de conservation.}

Dans cette expérience, la solution simple à base de $\mathrm{NaCl}$ et le MML,S sont utilisés comme dilueurs de conservation.

Conservation de la fécondabilité des ovules.

Les dilueurs à base de $\mathrm{NaCl}$ tamponnés avec du Tris ou du glycocolle à $0,02 \mathrm{M}$ conservent mietux la fécondabilité des ovtules que les solutions tamponnées avec un 
tampon minéral (carbonate-bicarbonate), au moins pendant les $\mathrm{I} 5$ premières minutes (fig. 2). Une dépression de fertilité apparaît entre la $2^{\mathrm{e}}$ et la $6^{\mathrm{e}}$ minute qui suit la mise en présence des ovules avec le dilueur quel que soit le tampon utilisé.

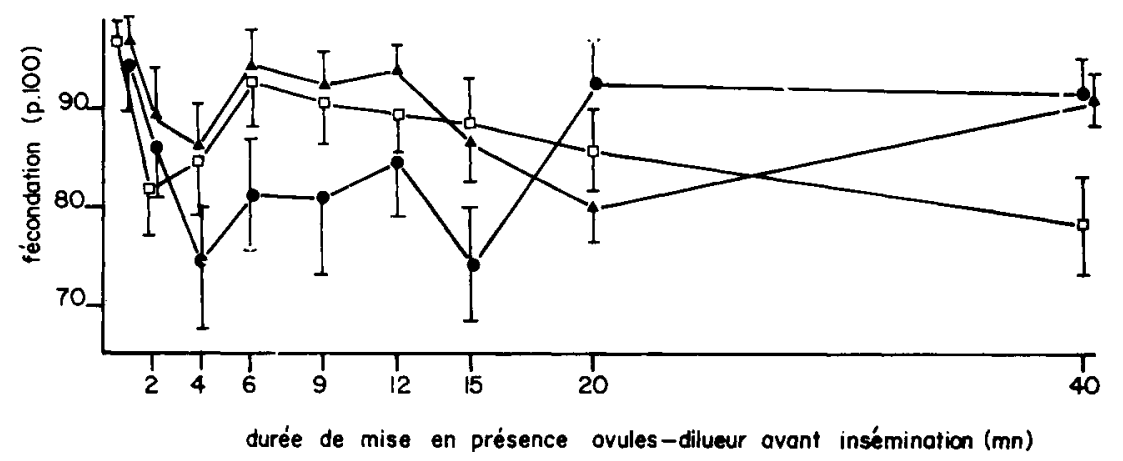

FIG. 2. - Effets de la nature du tampon ajouté au dilueur sur la conservation de la fécondabilité des ovules avant insémination

Tampons $0,02 \mathrm{M} \bullet$ Carbonate-bicarbonate

- Tris- $\mathrm{HCl}$

$\square$ Glycocolle

Dilueur : solution de $\mathrm{NaCl}$ posm 250, $\mathrm{pH} 9,0$

Taux de dilution final du sperme $:$ Io $^{-3}$

\section{Conservation du pouvoir fécondant du sperme.}

Le pouvoir fécondant des spermatozoïdes est mieux conservé lorsque le dilueur à base de $\mathrm{NaCl}$ est tamponné avec le glycocolle (tabl. 3). Au contraire, avec le MML,S

\section{TABLEAU 3}

Effet de la nature du tampon sur la conservation du pouvoir fécondant du sperme après mélange avec le dilueur

I_e protocole expérimental comporte le mélange du dilueur (Io $\mathrm{ml}$ de sol. $\mathrm{NaCl}$ posm $=250$ mosm) et du sperme (dilution finale $\left.=10^{-4}\right)$, pendant des temps variables $(30 \mathrm{~s}$ à $40 \mathrm{mn}$ ) avant l'addition des ovules.

Dans cette expérience la solution de $\mathrm{NaCl}$ est utilisée comme dilueur de conservation. (Pourcentage de fécondation après insémination immédiate $=98, \mathbf{r}$ p. I $о 0$ ).

\begin{tabular}{|c|c|c|c|c|c|c|c|c|c|}
\hline \multirow{2}{*}{$\begin{array}{l}\text { Tampon } \\
(0,02 \mathrm{M})\end{array}$} & \multirow{2}{*}{$\mathrm{pH}$} & \multicolumn{8}{|c|}{ p. 100 de fécondation après conservation du sperme dilué pendant } \\
\hline & & $30 \mathrm{~s}$ & $1 \mathrm{mn}$ & $2 \mathrm{mn}$ & $4 \mathrm{mnt}$ & $7 \mathrm{mn}$ & $10 \mathrm{mn}$ & $15 \mathrm{mn}$ & $40 \mathrm{mn}$ \\
\hline $\begin{array}{l}\text { Carbonate- } \\
\text { bicarbonate }\end{array}$ & 9,50 & 28,0 & 13,0 & 4,6 & 0,5 & 0 & 0 & 0 & 0 \\
\hline Tris- $\mathrm{HCl}$ & 9,52 & 21,2 & 6,4 & 1,5 & 1,0 & 0 & 0 & 0 & 0 \\
\hline $\begin{array}{l}\text { Glycocolle- } \\
\mathrm{NaOH}\end{array}$ & 9,32 & $5 \%, 9$ & 31,7 & 25,1 & 0,5 & 0,5 & 0,5 & 0 & 0 \\
\hline
\end{tabular}


l'utilisation du tampon Tris- $\mathrm{HCl}(\mathrm{o}, \mathrm{o} 2 \mathrm{M})$ permet une amélioration et une prolongation de la conservation du pouvoir fécondant par rapport au tampon glycocolle$\mathrm{NaOH}$ (tabl. 4),

\section{TABLEAU 4}

Pourcentage de fécondation d'ounles de Truite arc-en-ciel obtenu après dilution du sperme et conservation pendant 1 à 30 minutes dans un milieu minéral de liquide séminal (Io $\mathrm{ml}$ ) tamponné avec du Tris et du glycocolle 0,02 $\mathrm{M}$

(pourcentage de fécondation témoin obtenu après insémination dans solution de $\mathrm{NaCl}: 97,5 \mathrm{p} \bullet$ Ioo)

\begin{tabular}{|c|c|c|c|c|c|c|c|c|c|c|}
\hline \multirow{3}{*}{ Tampon } & \multirow{3}{*}{$\mathrm{pH}$} & \multicolumn{9}{|c|}{ Temps de mise en présence dilueur et sperme avant fécondation } \\
\hline & & \multicolumn{3}{|c|}{1 minute } & \multicolumn{3}{|c|}{15 minutes } & \multicolumn{3}{|c|}{30 minutes } \\
\hline & & $10^{-5}(1)$ & $10^{-4}$ & $10^{-3}$ & $10^{-5}$ & $10^{-4}$ & $11^{-3}$ & $10^{-5}$ & $10^{-4}$ & $10^{-3}$ \\
\hline \multirow{3}{*}{ Tris- $\mathrm{HCl}$} & 8,0 & 3,4 & 14,3 & 78,0 & $22, \ddot{2}$ & 5,4 & 33,1 & 4,5 & 5,5 & 37,3 \\
\hline & 8,5 & 16,6 & 60,8 & 82,8 & 51,6 & 40,3 & 65,8 & 15,6 & 33,7 & 66,7 \\
\hline & 9,0 & 16,4 & 88,2 & 89,7 & 31,1 & 41,6 & 80,9 & 24.8 & 29,1 & 71,1 \\
\hline \multirow{4}{*}{$\begin{array}{c}\text { Glycocolle- } \\
\mathrm{NaOH}\end{array}$} & 9,0 & 8,3 & 54,3 & 92,0 & 34,1 & $\$ 1,2$ & 67,5 & $9, \mathbf{1}$ & 27,0 & 36,5 \\
\hline & 9,5 & 7,0 & 74,8 & 79,6 & 28,3 & 22,3 & 71,7 & 12,2 & 36,8 & 19,4 \\
\hline & 10,0 & 14,0 & 60,5 & 77,6 & 11,8 & 12,7 & 64,9 & 11,5 & 20,8 & 21,4 \\
\hline & & & & & & & & & & \\
\hline
\end{tabular}

(1) Taux de dilution du sperme.

B. - Variation du pH du dilueur d'insémination après introduction expérimentale de $\mathrm{NaOH}$ ou $\mathrm{HCl}$, de liquide colomique et d'ovules

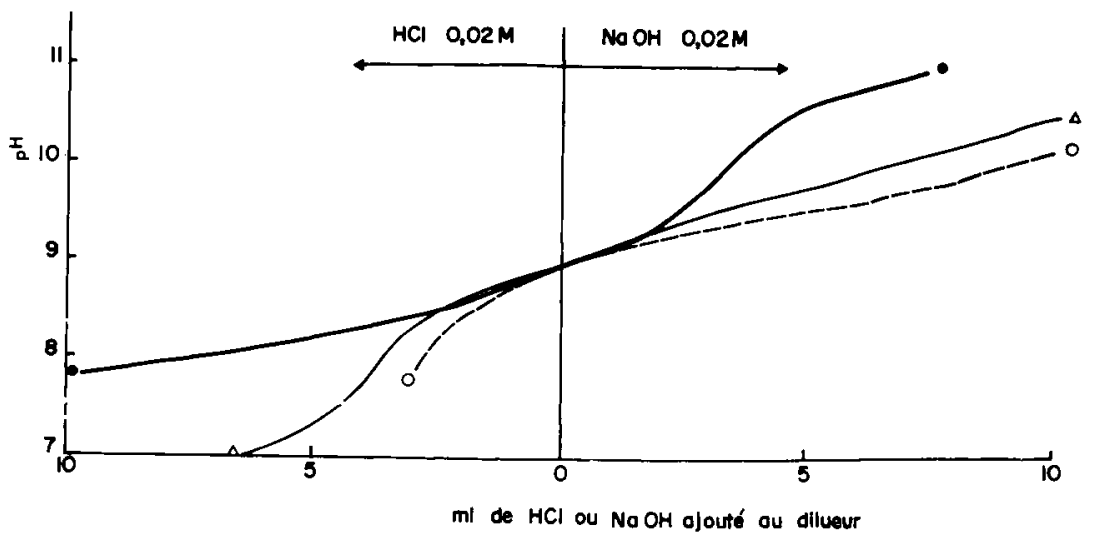

FIG. 3. - Variations $d u p H d u$ dilueur d'insémination

(solution simple de $\mathrm{NaCl}$ tamponnée, pression osmotique finale $250 \mathrm{mosm}, \mathrm{pH} 9,0$ ) après addition de volumes croissant de $\mathrm{NaOH}$ ou $\mathrm{HCl} 0,02 \mathrm{M}$

Volume du dilueur $=20 \mathrm{ml}$

Tampons $(0,02) \Delta$ Carbonate-bicarbonate

- Tris- $\mathrm{HCl}$

- Glycocolle- $\mathrm{NaOH}$ 
Après introduction de quantités croissantes de $\mathrm{NaOH}$ ou de $\mathrm{HCl} 0,02 \mathrm{M}$, le pH du dilueur varie de façon importante quel que soit le tampon testé (fig. 3). A la concentration utilisée $(0,02 \mathrm{M})$ ces tampons n'assurent donc pas une stabilité remarquable du $\mathrm{pH}$ du dilueur. L'introduction de liquide colomique ou d'ovules (fig. 4) montre cependant que cette stabilité reste suffisante pour maintenir le $\mathrm{pH}$ dans une zone favorable. En effet, le volume de liquide cœlomique $(\mathrm{pH} 8, \mathrm{I2})$ à introduire pour diminuer de 0,2 unité le $\mathrm{pH}$ d'un volume de $20 \mathrm{ml}$ de dilueur est de II $\mathrm{ml}$ avec l'acide borique- $\mathrm{NaOH}$, de Io $\mathrm{ml}$ avec le carbonate-bicarbonate, de $8,5 \mathrm{ml}$ avec le Tris- $\mathrm{HCl}$ et de $4,5 \mathrm{ml}$ avec le glycocolle- $\mathrm{NaOH}$ (fig. 4 a). Après introduction de quantités croissantes d'ovules, les tampons carbonate-bicarbonate et Tris stabilisent beaucoup mieux le $\mathrm{pH}$ du dilueur que le glycocolle- $\mathrm{NaOH}$ (fig. 4 b).
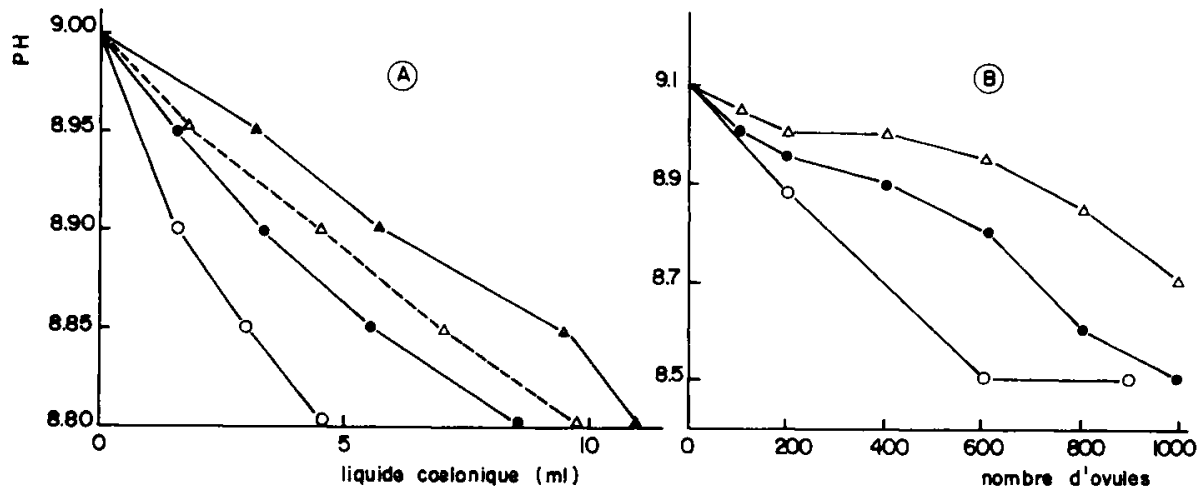

FIG. 4. - Variations $d u$ pH du dilueur d'insémination de même caractéristique que dans la fig. 3 . après addition de liquide colomique (A) et d'ovules (B)

- Borate- $\mathrm{NaOH}(0,02 \mathrm{M})$

\section{DISCUSSION E'T CONCLUSION}

\section{A. - Choix de la nature et de la molarité du tampon du dilueur d'insémination}

L'amélioration du pourcentage de fécondation qui résulte de l'emploi, lors de l'insémination, de dilueurs tamponnés à un $\mathrm{pH}$ relativement élevé semble pouvoir être mis en relation avec l'augmentation de l'intensité et de la durée de motilité des spermatozoïdes dans la zone de $\mathrm{pH} 9$ et 9,5 (BILLARD et al., données non publiées). Également chez la Truite arc-en-ciel et en prenant pour critère la motilité des spermatozoïdes. INABA et al. (I958) observent une activité dans une gamme de pH très large (entre 4,5 et 10,4 ) mais les mouvements seraient plus actifs dans la zone basique. L'effet favorable des $\mathrm{pH}$ alcalins a aussi été signalé sur l'attraction chimiotactique des spermatozoïdes vers le micropyle de l'œuf chez la Bouvière (Suzuki, I960). Chez le Mulet (Mugil cephalus) la durée de motilité des spermatozoïdes semble être maximum à un taux voisin de la neutralité (HINEs et YASHOUV, I97I).

I1 est done important de disposer d'un dilueur d'insémination dont le $\mathrm{pH}$ reste stable après introduction des gamètes. L'utilisation de tampons à $0,02 \mathrm{M}$ dans le di- 
lueur d'insémination n'assure pas une stabilité parfaite du $\mathrm{pH}$ du milieu ainsi que le montre l'introduction expérimentale de $\mathrm{NaOH}$ et $\mathrm{HCl}$, de liquide coelomique ou d'ovules dans le milieu. Cette chute de $\mathrm{pH}$ peut être réduite par l'emploi d'un tampon à $\mathrm{o}, \mathrm{I} \mathrm{M}$, mais dans ce cas, au moins pour les tampons carbonate-bicarbonate et glycocolle, le pourcentage de fécondation s'en trouve affecté. Le doublement du volume de dilueur utilisé lors de l'insémination permet de limiter la chute de $\mathrm{pH}$, tout en conservant un tampon à $0,02 \mathrm{M}$. Il en résulte même aux fortes dilutions $\left(\mathrm{IO}^{-4}\right)$ une amélioration du pourcentage de fécondation qui peut cependant être due à l'augmentation du nombre de spermatozoïdes (ce dernier reste constant par unité de volume de dilueur, mais le nombre théorique de spermatozoïes par ovule s'est trouvé augmenté). Toutefois, dans les conditions de la pratique, il est au contraire préférable de limiter le volume de dilueur afin de réduire la quantité de sperme et de respecter un certain rapport entre la quantité d'ovules et le volume de dilueur (BILLARD et al., I974).

Du fait que le pourcentage de fécondation subsiste à un niveau maximum dans une gamme de $\mathrm{pH}$ relativement large - entre 8,5 et 9,5 (fig. I), il reste préférable de tamponner le dilueur à un $\mathrm{pH}$ compris entre 9,2 et 9,5 avec un tampon $0,02 \mathrm{M}$; dans ces conditions, la chute de $\mathrm{pH}$ d'environ 0,2 unité qui suit l'introduction des ovules dans le dilueur, ne risque pas de porter le $\mathrm{pH}$ du mélange hors de la zone favorable.

Quant au choix du tampon, pour les taux de dilution conseillés en pisciculture $\left(\mathrm{IO}^{-3}\right.$ à $\left.\mathrm{IO}^{-2}\right)$, chacun des tampons testés dans cette étude pourrait, en se référant aux pourcentages de fécondation obtenus, être retenus pour stabiliser le $\mathrm{pH}$ de la solution de $\mathrm{NaCl}$ à posm $25^{\circ}$ proposée comme dilueur d'insémination. Le système borate- $\mathrm{NaOH}$ qui a le meilleur pouvoir tampon a cependant un effet dépressif sur le taux de fécondation. Le tampon carbonate-bicarbonate, bien qu'entraînant, aux fortes dilutions, une diminution du pourcentage de fécondation pour les $\mathrm{pH}<9,5$, se révéle pour les $\mathrm{pH}$ compris entre 9 et 9,4 aussi satisfaisant que les tampons orga. niques et présente en outre l'avantage de mieux stabiliser le $\mathrm{pH}$ du dilueur. Le tampon carbonate-bicarbonate à $0,02 \mathrm{M}$ est actuellement celui que nous retenons pour tamponner le dilueur d'insémination mais l'emploi de tampons organiques n'est pas à exclure.

\section{B. - Choix du tampon du dilueur de conservation}

Des solutions de $\mathrm{NaCl}$ tamponnées avec Tris- $\mathrm{HCl}$, glycocolle- $\mathrm{NaOH}$ ou carbonate-bicarbonate peuvent constituer de bons milieux de conservation des ovules. Par contre le pouvoir fécondant du sperme n'est pas maintenu bien que l'utilisation du tampon glycocolle-NaOH permette, après 2 minutes, d'obtenir davantage de fécondations qu'avec les autres tampons testés (tabl. 3). Cela pourrait correspondre à un effet protecteur au moins temporaire de l'acide aminé sur les spermatozoïdes.

La conservation du pouvoir fécondant du sperme est optimum lorsque le MMLS est tamponné à un $\mathrm{pH}$ de $9, \mathrm{o}$. Avec ce milieu et à la différence des dilueurs à base de $\mathrm{NaCl}$ précédents, le tampon Tris- $\mathrm{HCl}$ se révèle supérieur au tampon glycocolle- $\mathrm{NaOH}$.

En conclusion, le MMLS qui assure une bonne conservation du pouvoir fécondant à la fois des ovules et des spermatızoïdes peut être tamponné avec du Tris- $\mathrm{HCl}$ o,02 M à un $\mathrm{pH}$ de $9, \mathrm{o}$.

Ces techniques de dilution dans des solutions salines simples qui entraînent une 
immobilisation rapide des spermatozoïdes et qui n'ont jamais été retenues chez les Mammifères sont cependant compatibles avec le mode de fécondation externe chez la Truite et aboutissent, dans le cas du dilueur d'insémination, à des taux de fécondation très satisfaisants, en général plus élevés qu'après insémination selon la méthode sèche.

Reçu pour publication en mars 1974.

\section{REMERCIEMENTS}

M. Bonicel a participé à la conduite des incubations. La frappe du manuscrit est due à Mme Marcel.

\section{SUMMARY}

\section{ARTIFICIAL, INSEMINATION OF THE TROUT (SALMO GAIRDNERI RICHARDSON). III. - DEFINITION OF THE NATURE AND MOLARITY OF THE BUFFER TO USE WITH INSEMINATION AND CONSERVATION DILUENTS}

In experiments to determine the nature and molarity of the buffer to use with two previously defined insemination and conservation diluents, the effects of different buffers on diluent $\mathrm{pH}$ stability are tested after introduction of increasing quantities of $\mathrm{NaOH}$ and $\mathrm{HCl}$, cœlomic fluid and ovules. We also tested effects of these buffers on the percentage of fertilization obtained in small lots of 200 ovules. The following results are observed :

I. The insemination diluent (solution of $\mathrm{NaCl}$ at $25^{\circ}$ mosm) may be favorably buffered with carbonate-bicarbonate buffer $0.02 \mathrm{M}, 9.2-9.5 \mathrm{pH}$. This buffer is more stable after in troduction of a large quantity of ovules than the other buffers tested (Tris- $\mathrm{HCl}$, Glycocolle- $\mathrm{NaOH}$ ), but fertility rate is the same with each of these buffers.

2. When the mineral medium of the seminal fluid (MMLS) is used as a conservation diluent, conservation of sperm fertilizing ability is better when the buffer used is Tris-HCl ; optimum $\mathrm{pH}$ is about 9.0 .

\section{RÉFERENCES BIBLIOGRAPHIQUES}

Billard R., Petit J., Jalabert B., Szollosi D., ig73. Artificial insemination in trout using a sperm diluant. Symp. Early Life History Fish, OBan, Blaxter Edit., 7I5-723.

BILlard R., JalaberT B., I974. L'Insémination artificielle de la Truite (Salmo gaidneri RICHARDSON). II. Comparaison des effets de différents dilueurs sur la conservation du pouvoir fécondant des gamètes avant insémination. Ann. Biol. anim. Bioch. Biophys., 14, 6ог-6ro.

Billard R., Petit J., Chevassus B., I974. V. Définition du rapport gamètes/dilueur optimum lors de l'insémination (en préparation).

Delory, King, I945. Biochem. J., 39, 245. Cité par Gomori, I955.

Gomori G., 1955. Preparation of buffers for use in enzyme studies, I38-146 in Colowick and Kaplan. Methods in Enzymology, 1, Acad. Press NY.

Hines R., Yashouv A., I97I. Some environmental factors influencing the activity of spermatozoa of Mugil capito cuvier, a grey mullet. J. Fish Biol., 3, I23-127.

Inaba D., Nomura M., Suyama M., I958. Studies on the improvement of artificial propagation in trout culture. II. On the $\mathrm{pH}$ values of eggs, colomic fluid and others. Bull. Jap. Soc. Sci. Fish., 23, $762-765$.

Kohler R., i971. Buffer solutions, 280-282; in Scientific Tables, Dier and Letner, Ciba Geigy, Basel. 
Petit J., Jalabert B., Chevassus B., Billard R., i973. L'Insémination artificielle de la Truite (Salmo gairdneri RICHARDSON). I. Effets du taux de dilution du pH et de la pression osmotique du dilueur sur la fécondation. Ann. Hydrobiol., 4, 201-210.

Sörensen S. P. L., I909. Biochem. Z., 21, I3I-304. Cité par Gomori, I955.

SUzUKI R., I960. Sperm activation and aggregation during fertilization in some fishes. IV. Effects of $\mathrm{pH}$, heat and other agents upon sperm-stimulating factor. Jap. J. Zool., 12, 465-476. 